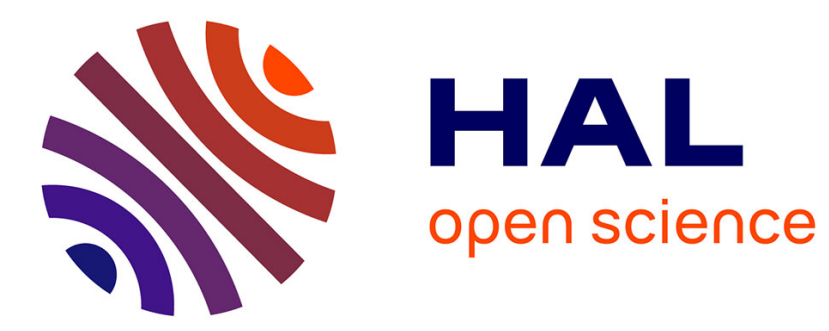

\title{
Mixing in superconducting weak links : numerical calculations and experimental results
}

\author{
F. Auracher, T. van Duzer
}

\section{To cite this version:}

F. Auracher, T. van Duzer. Mixing in superconducting weak links: numerical calculations and experimental results. Revue de Physique Appliquée, 1974, 9 (1), pp.233-241. 10.1051/rphysap:0197400901023300 . jpa-00243746

\section{HAL Id: jpa-00243746 https://hal.science/jpa-00243746}

Submitted on 1 Jan 1974

HAL is a multi-disciplinary open access archive for the deposit and dissemination of scientific research documents, whether they are published or not. The documents may come from teaching and research institutions in France or abroad, or from public or private research centers.
L'archive ouverte pluridisciplinaire HAL, est destinée au dépôt et à la diffusion de documents scientifiques de niveau recherche, publiés ou non, émanant des établissements d'enseignement et de recherche français ou étrangers, des laboratoires publics ou privés. 


\title{
MIXING IN SUPERCONDUCTING WEAK LINKS : NUMERICAL CALCULATIONS AND EXPERIMENTAL RESULTS
}

\author{
F. AURACHER $(*)$ and T. VAN DUZER \\ Department of Electrical Engineering and Computer Sciences \\ and the Electronics Research Laboratory \\ University of California, Berkeley, California 94720, USA
}

\begin{abstract}
Résumé. - Nous reportons ici les calculs numériques que nous avons faits pour déterminer les propriétés des contacts faibles supraconducteurs utilisés comme mélangeurs avec un oscillateur local externe (LO). Nos calculs sont réalisés à partir d'un modèle de jonction shuntée par une résistance. Nous supposons que l'oscillateur local et le signal sont des sources idéales d'amplitude $I_{\mathrm{LO}}$ et $I_{\mathrm{S}}$ et de pulsation $\omega_{\mathrm{LO}}$ et $\omega_{\mathrm{S}}$. Nous calculons la tension IF aux bornes du contact faible en fonction du courant de polarisation, de l'amplitude du courant $I_{\mathrm{LO}}$ et de la pulsation normalisée $\Omega_{\mathrm{s}}=\hbar \omega_{\mathrm{s}} /\left(2 \mathrm{e} I_{\mathrm{c}} R\right)$ où $I_{\mathrm{c}}$ est le courant critique et $R$ la résistance shunt. Si les rapports $\omega_{\mathrm{IF}} / \omega_{\mathrm{LO}}$ et $I_{\mathrm{s}} / I_{\mathrm{c}}$ sont petits, nous trouvons une réponse IF linéaire. En utilisant cette méthode nous avons calculé la variation de la réponse IF en fonction de la fréquence, estimé le rendement de conversion dans le cas de l'adaptation en présence et en l'absence de bruit, enfin nous avons calculé la puissance de l'oscillateur local requise. Nous avons fabriqué des microponts en utilisant un faisceau d'électrons pour le masquage. Nous avons réalisé des microponts de $2000 \AA$ de large et de $1000 \AA$ de long dans des films d'indium d'épaisseur $1000 \AA$ et avons étudié leurs caractéristiques $I-V$ en présence d'un rayonnement et leurs propriétés de mélange en bande $X$. Nous avons mesuré une puissance équivalente de bruit de $10^{-13} \mathrm{~W} / \mathrm{Hz}^{1 / 2}$ et un domaine dynamique de $23 \mathrm{~dB}$ (écart de $3 \mathrm{~dB}$ de la linéarité) et estimé qu'avec une adaptation optimum, il serait possible d'atteindre respectivement $3 \times 10^{-18} \mathrm{~W} / \mathrm{Hz}^{1 / 2}$ et $74 \mathrm{~dB}$.
\end{abstract}

Abstract. - We report here numerical calculations on the properties of superconducting weak links as mixers with external local oscillators (LO). Our calculations are based on the resistively shunted-junction model. We assume that the LO and the signal are ideal current sources of amplitudes $I_{\mathrm{LO}}$ and $I_{\mathrm{S}}$ and of frequencies $\omega_{\mathrm{LO}}$ and $\omega_{\mathrm{s}}$, respectively. We calculate the IF voltage across the weak link as a function of the bias current, the LO current amplitude $I_{\mathrm{LO}}$, and the normalized signal frequency $\Omega_{\mathrm{s}}=\hbar \omega_{\mathrm{s}} /\left(2 \mathrm{e} I_{\mathrm{c}} R\right)$, where $I_{\mathrm{c}}$ is the critical current and $R$ is the shunt resistance. If $\omega_{\mathrm{IF}} / \omega_{\mathrm{LO}}$ and $I_{\mathrm{s}} / I_{\mathrm{c}}$ are small, we find a linear IF response. Using this method we find the frequency dependence of the IF response, estimate the conversion efficiency for the matched case in both the absence and the presence of noise, and calculate the required LO power. We have fabricated thinfilm constrictions by using electron lithography. We have made constrictions of indium films with thicknesses of $\approx 1200 \AA$ with widths as narrow as $2000 \AA$ and lengths as short as $1000 \AA$ and have studied their $I-V$ characteristics in the presence of RF radiation and measured their $\mathrm{X}$-band mixing properties. We have measured an $N E P$ of $10^{-13} \mathrm{~W} / \mathrm{Hz}^{1 / 2}$ and a dynamic range of $23 \mathrm{~dB}$ ( $3 \mathrm{~dB}$ deviation from linearity) and estimate that, with optimum matching, these would become $3 \times 10^{-18} \mathrm{~W} / \mathrm{Hz}^{1 / 2}$ and $74 \mathrm{~dB}$, respectively.

1. Introduction. - Josephson devices have been used in mixing experiments since the late 1960s. The first extensive report was that of Grimes and Shapiro [1] in which they reported the mixing of signals in the frequency ranges around $23 \mathrm{GHz}$ and $72 \mathrm{GHz}$ using superconducting point contacts. They analyzed the mixing process assuming voltage sources for the two signals. Since that time it has become clear that,

(*) Present address : Research Labs of Siemens AG, D 8 Munich 70, Hofmannstr. 51, W. Germany.

Research sponsored by the US Army Research Office, Durham, Grant DA-ARO-D-31-124-70-G 60. because Josephson devices typically have very low impedances $(\approx 1 \Omega)$, it is a better approximation to assume current sources. We report here the result of numerical calculations of mixing for a model of the Josephson device in which it is assumed that the ideal Josephson device is shunted by a constant resistance and is driven by constant current sources.

Anderson and Dayem [2] first reported RF measurements on constricted thin films and more extensive measurements were reported subsequently by Dayem and Wiegand [3]. The heights of RF induced steps and their dependence on the RF amplitude have been studied experimentally in recent work [4] and these 
have been compared with analog-computer calculations based on the same simple resistively shunted junction model as is used in this paper. The constricted thin films reported so far have been made either by deposition of the metal vapor through shadow masks or by scribing thin films. The latter technique has sometimes resulted in constrictions with very pronounced step structure that fits quite well the theoretically predicted dependence on RF power.

We report here on step-height and mixing measurements using constrictions made by electron lithography. A computer-controlled scanning electron microscope is used to expose a pattern in an electron resist in the form shown in figure 1. Upon development, the

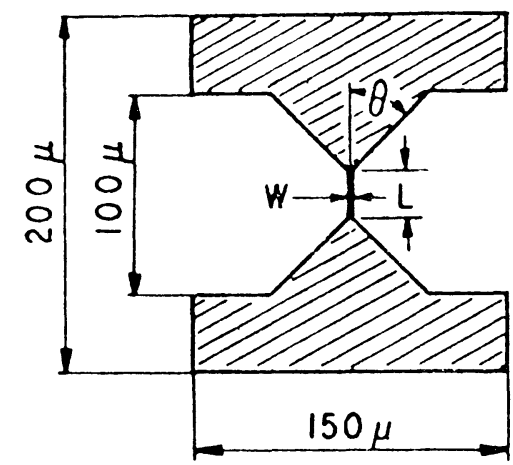

FIG. 1. - Shape of constriction fabricated by electron lithography.

exposed portion of the resist is removed as in figure $2 a$. Then a vapor deposition of the superconductor produces the result shown in figure $2 b$; the metal film is on the substrate in the pattern of the constriction and on top of the remaining resist elsewhere. As a result of the undercutting of the resist, there is no connection between the two regions of metal so upon

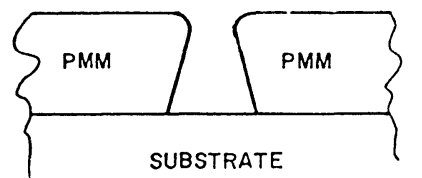

AFTER DEVELOPMENT

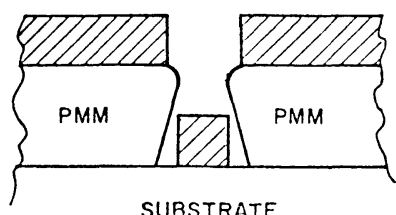

AF TER

METAL DEPOSIT

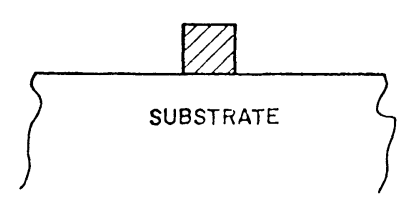

DESIRED METAL PATTERN ON SUBSTRATE AFTER RESIST REMOVAL

FIG. 2. - Steps in device fabrication by electron lithography. dissolving the remaining resist, the metal film outside the constriction pattern is removed as shown in figure $2 c$. The device remains on the substrate. Figure 3 shows a device typical of ones we made ; the process is coming under increasingly accurate control but so far we have not been able to get constrictions to predetermined dimensions with an accuracy of better than about $1000 \AA$.

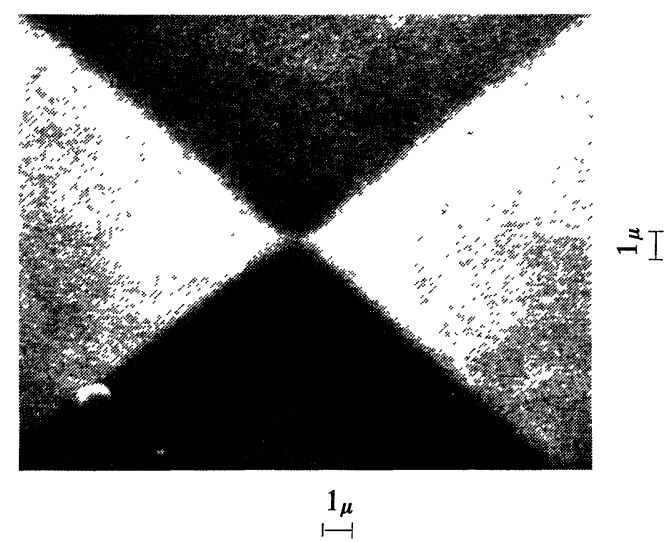

FIG. 3. - Electron micrograph of a finished indium constriction.

We have made numerical calculations on the simple resistively shunted junction, the equivalent circuit of which is shown in figure 4 . The signals and bias are assumed to be applied by current sources and capacitance is neglected. Earlier analysis of this model done by analog computation [5] yielded information about the dependence of RF induced step structure on the RF amplitude. Our calculations have been directed at quantities of direct importance to the mixing application. For the most part, we assumed that the intermediate frequency is much smaller than the signal frequency as this covers most of the practical cases and the computations are thereby simplified. We calculate the frequency dependence of the IF response, linearity (and, hence, dynamic range), conversion efficiency, and the required local oscillator power.

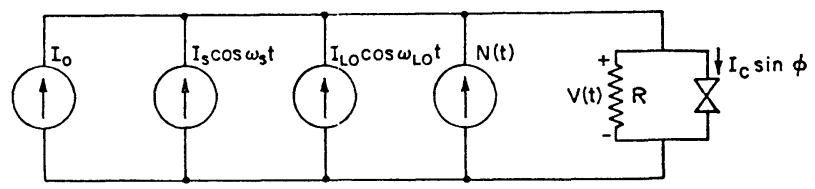

FIG. 4. - Equivalent circuit for the resistively shunted junction.

An important parameter for mixing with Josephson devices is the normalized signal frequency

$$
\Omega_{\mathrm{s}}=\hbar \omega_{\mathrm{s}} / 2 \mathrm{e} I_{\mathrm{c}} R
$$

where $\omega_{\mathrm{s}}$ is the signal angular frequency, $I_{\mathrm{c}}$ is the critical current, and $R$ is the shunt resistance (Fig. 4). For a given Josephson device, $\Omega_{\mathrm{s}}$ can be determined quickly from measurements of the zeros in the dependence of the RF induced steps on RF amplitude if 
that dependence fits the model. We have applied the test to point contacts and thin-film constrictions, with a better fit for the former.

Test were made on a number of constrictions of varying sizes and of both indium and vanadium. We were unable to fabricate the vanadium constrictions having lengths and widths of the constricted region less than about $6000 \AA$ and were unable to observe RF step structure on ones that large. This we take to be further evidence of the idea that the constriction dimensions must be comparable with the coherence length (about $500 \AA$ in vanadium). Laibowitz [6] has recently made niobium constrictions having dimensions of the order of $3000 \AA$ in which he could see RF-induced step structure. All of our mixing measurements were made on indium constrictions; the results are presented in Section 3.

2. Mixing calculations. - Calculations were made for the model shown in figure 4 with main emphasis on the practical case where the intermediate frequency is much smaller than the signal frequency and the signal current amplitude is much smaller than the local oscillator current. In this case the sum of the local oscillator and signal currents has the form of a wave at the local oscillator frequency which is amplitude modulated at the intermediate frequency. The value of the RF amplitude determines the height of the step at zero voltage ; since the former changes at the intermediate frequency, so does the latter. These changes of the zero-voltage step height result in a modulation of the voltage across the device as illustrated in figure 5. In this case we can find the IF amplitude from a knowledge of the dependence of the zerovoltage step height on the amplitude of a single RF signal; this is how most of our calculations were made. In an earlier publication [7], we showed numerical results of calculations using the complete model in figure 4 and the small-signal, low-intermediate-frequency approximation discussed above ; the comparison showed very good agreement.

The situation illustrated in figure 5 assumes an infinite IF load impedance and gives an IF voltage $V_{\mathrm{IF}^{\infty}}$. If we take account of a finite IF load resistance $R_{\mathrm{IF}}$ and assume for simplicity that it is ohmic at the IF frequency but infinite at high frequencies, then the IF voltage output is

$$
V_{\mathrm{IF}}=V_{\mathrm{IF} \infty} \frac{\rho}{1+\rho}
$$

where $R_{\mathrm{IF}}=\rho R_{\mathrm{dyn}}$ and $R_{\mathrm{dyn}}$ is the reciprocal of the slope of the $I-V$ characteristic at the point of observation and is assumed to be constant.

2.1 Conversion EFFICIENCY. - We have shown in reference [7] that the conversion efficiency can be found for this model to be

$$
C_{\mathrm{e}}=\frac{\rho}{(1+\rho)^{2}}\left(\frac{\partial I_{0}}{\partial I_{\mathrm{RF}}}\right)^{2} \frac{R_{\mathrm{dyn}}}{R_{\mathrm{s}}}
$$

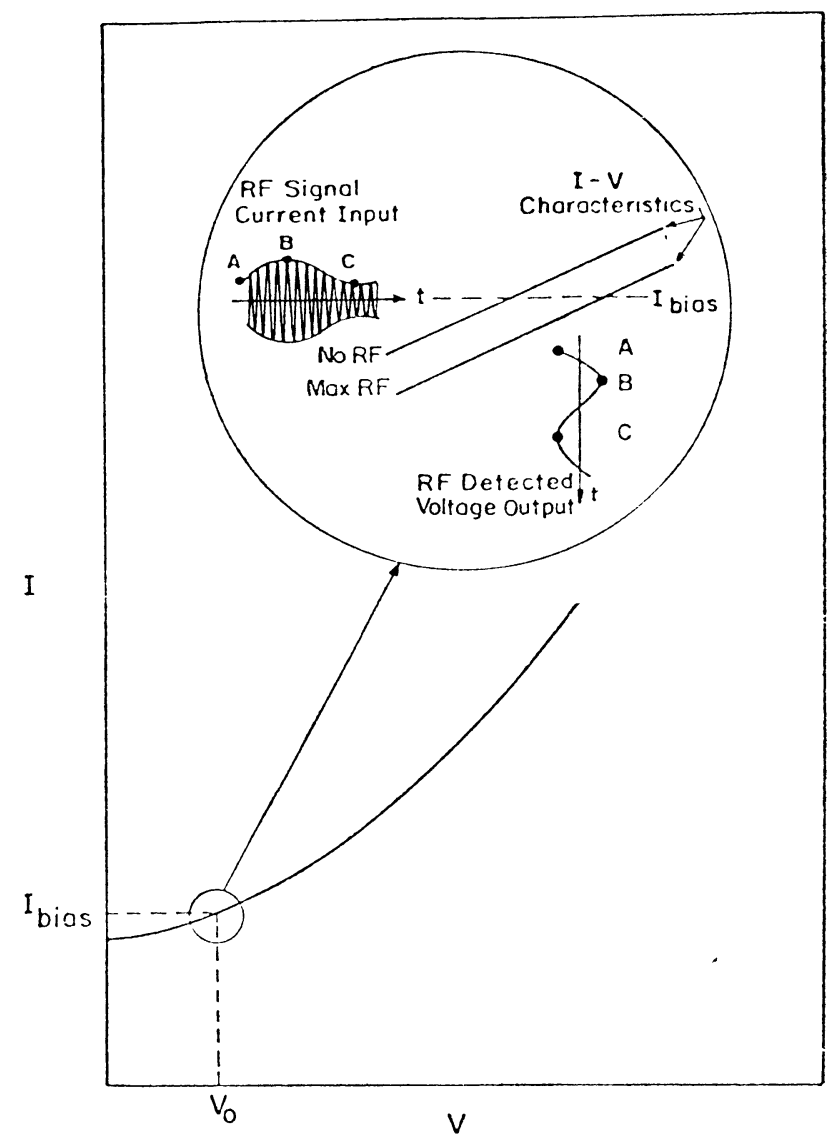

FIG. 5. - Detection of a modulated RF signal with a resistively shunted Josephson device.

where $\partial I_{0} / \partial I_{\mathrm{RF}}$ measures the sensitivity of the zerovoltage step height $I_{0}$ to the RF signal at the operating point $I_{\mathrm{LO}}$. The conversion efficiency is maximized by matching the IF load to the dynamic resistance $(\rho=1)$, by operating at the point of largest variation of $I_{0}$ with $I_{\mathrm{RF}}$, and by having a high ratio of dynamic resistance $R_{\mathrm{dyn}}$ to the impedance of the device at the signal frequency, $R_{\mathrm{s}}$.

We discuss now the important mixing parameters in more detail. The dynamic resistance depends on both the LO current $I_{\text {LO }}$ and the normalized frequency $\Omega_{\text {LO }}$. For small normalized frequencies and optimum LO current, the dynamic resistance becomes very high and is essentially only noise-limited, whereas for high normalized frequencies it has a low value $\left(R_{\mathrm{dyn}} \sim R\right)$ and is insensitive to noise.

In figure $6 a$ we show the square of the maximum rate of change of the critical current with applied RF current versus normalized frequency, as calculated for the RSJ model. This parameter approaches 1.0 asymptotically for $\Omega_{\mathrm{RF}} \rightarrow 0$ and rolls off like $\left(0.581 / \Omega_{\mathrm{RF}}\right)^{2}$ for large $\Omega_{\mathrm{RF}}$ as predicted by the voltagesource model. This shows the importance of working at low normalized frequency

$$
\left(\Omega_{\mathrm{s}}=\frac{\hbar \omega_{\mathrm{s}}}{2 \mathrm{e} I_{\mathrm{c}} R}<1\right),
$$


i. e., for a given signal frequency $\omega_{\mathrm{s}}$ we want $I_{\mathrm{c}} R$ to be larger than $\hbar \omega_{\mathrm{s}} / 2 \mathrm{e}$. However, theory and experiments show that $I_{\mathrm{c}} R$ is related to the energy gap [8], by the equation:

$$
I_{\mathrm{c}} R=\frac{\pi}{4 \mathrm{e}} \frac{\Delta^{2}(T)}{k T_{\mathrm{c}}} .
$$

Therefore, one should have $\omega_{\mathrm{s}}<\pi \Delta^{2}(T) / 2 \hbar k T_{\mathrm{c}}\left({ }^{1}\right)$ which for zero temperature is about $1050 \mathrm{GHz}$ for $\mathrm{Nb}$ and $360 \mathrm{GHz}$ for In. One should, therefore, use high $T_{\mathrm{c}}$ materials and work at low reduced temperatures in order to obtain a low normalized frequency. A limitation, at least in the case of constricted thin films, is that they usually do not behave according to the RSJ model and have low detection sensitivity at low temperatures unless their dimensions are very small.

We note that this mode of mixing is very similar to RF (video) detection with weak links, a difference being that in the case of mixing we can adjust the bias current and the LO power so we can operate in the region of both high dynamic resistance and a large $\partial I_{\mathrm{c}} / \partial I_{\mathrm{RF}}$. (In the case of video-detection the output depends on

$$
\left.\left.\frac{\hat{\sigma}^{2} I_{\mathrm{c}}}{\partial I_{\mathrm{RF}}^{2}}\right|_{I_{\mathrm{RF}}=0} \quad \text { rather than }\left.\quad \frac{\partial I_{\mathrm{c}}}{\partial I_{\mathrm{RF}}}\right|_{I_{\mathrm{LO}}}\right) .
$$

Of course, the output is at $\omega_{\mathrm{IF}}$ rather than dc, allowing for more sensitive amplifiers, in the case of mixing.

In reference [7] we showed the dependence of conversion efficiency on the local-oscillator current and the normalized signal frequency. It was seen that the highest conversion efficiencies occur where the localoscillator current $I_{\text {LO }}$ reduces the zero-voltage step $I_{0}$ to one half its value with zero $I_{\mathrm{LO}}$. This is the steepest part of the $I_{0}$ vs $I_{\text {LO }}$ relation (highest $\partial I_{0} / \partial I_{\mathrm{LO}}$ in eq. (2)). It is further seen that the maximum conversion efficiency occurs for the lowest normalized signal frequencies. With $\Omega_{\mathrm{s}}=0.4$ there was a conversion gain in excess of 4 without noise ; the calculation with noise corresponding to Johnson noise in the shunt resistance (assumed effective $T=4 \mathrm{~K}$ and $I_{\mathrm{c}}=100 \mu \mathrm{A}$ ) showed a reduction of the maximum conversion efficiency to a little greater than unity. This is a result of a reduction of the dynamic resistance which is especially prominent at low $\Omega_{\mathrm{s}}$. If a finite source impedance is used, the source impedance must be taken into account in evaluating $\Omega_{\mathrm{s}}$. Conversion efficiencies as high as 4.0 have recently been observed by Taur et al. [9] for point contacts at $36 \mathrm{GHz}$; the normalized signal frequency in that work was 0.5 taking account of the source impedance.

(1) This condition can be written as $\hbar \omega_{\mathrm{s}}<2.74 \Delta(0)$ for $T=0$, i. e., the signal frequency should be smaller than the energy gap frequency.

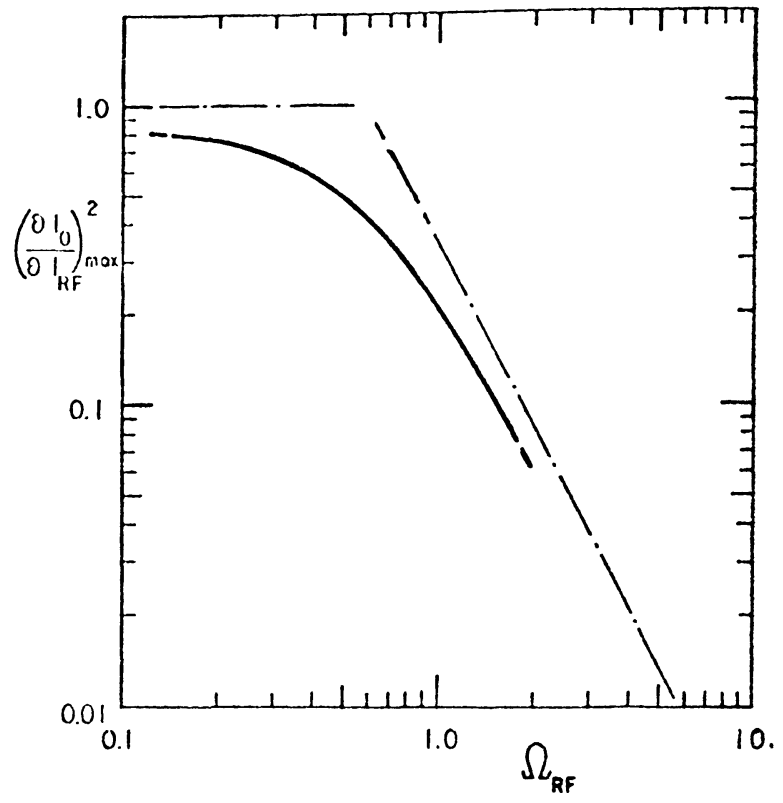

FIG. $6 a$. - The numerically calculated parameter $\left(\partial I_{0} / \partial I_{\mathrm{RF}}\right)_{\max }^{2}$ which is important for the conversion efficiency of the mixer, as a function of the normalized RF frequency $\Omega_{\mathrm{RF}}$.

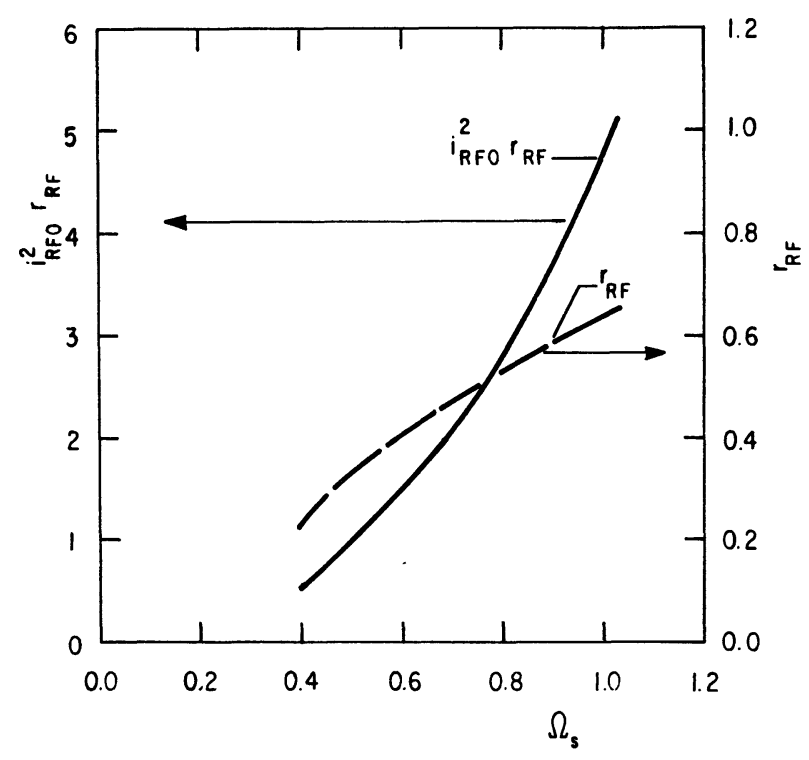

FIG. $6 b$. - Calculated normalized RF impedance $r_{\mathrm{RF}}=\boldsymbol{R}_{\mathrm{RF}} / \boldsymbol{R}$ of the weak link (when biased at $V_{0} \approx \frac{1}{2} \hbar \omega_{\mathrm{LO}} / 2 \mathrm{e}$ ) seen by the LO and the quantity $i_{\mathrm{RFO}}^{2} r_{\mathrm{RF}}$ which is proportional to the LO power as a function of the normalized RF frequency $\Omega_{\mathrm{s}}$.

2.2 LOCAL OSCILLATOR POWER REQUIRED FOR MIXING. - The LO power $P_{\text {LO }}$ in the weak link with RF impedance $R_{\mathrm{RF}}$ can be written as

$$
P_{\mathrm{LO}}=\frac{I_{\mathrm{LO}}^{2}}{2} R_{\mathrm{RF}}=\frac{1}{8}\left(I_{\mathrm{c}} R\right) I_{\mathrm{c}} i_{\mathrm{RFO}}^{2} r_{\mathrm{RF}}
$$

where $r_{\mathrm{RF}}=R_{\mathrm{RF}} / R$.

Taking $R_{\mathrm{RF}}$ for $I_{\mathrm{LO}}=0.5 I_{\mathrm{RFO}}$, where $I_{\mathrm{RFO}}$ is the RF current amplitude that depresses the critical current to zero (i. e., the LO current amplitude for which the conversion efficiency is maximum) and for a dc bias 
in the center between the 0th and 1st RF induced step, we find that the LO power required is proportional to the critical current and depends on the normalized frequency. Figure $6 b$ shows $i_{\mathrm{RFO}}^{2} r_{\mathrm{RF}}$ and $r_{\mathrm{RF}}$ as functions of the normalized frequency. Assuming a normalized frequency $\Omega_{\mathrm{s}}=0.39$, a critical current of $100 \mu \mathrm{A}$, and $R=1 \Omega$ (which are typical values for constrictions operated near the transition temperature), the LO power required for maximum conversion efficiency is then $P_{\mathrm{LO}} \approx 6 \times 10^{-9} \mathrm{~W}$. As

$$
\Omega_{\mathrm{s}}=\hbar \omega_{\mathrm{s}} / 2 \mathrm{e} I_{\mathrm{c}} R,
$$

the actual RF frequency for the above assumptions would be $\omega_{\mathrm{s}} / 2 \pi \approx 50 \mathrm{GHz}$. The extremely low LO power required for this type of mixer may prove to be important in some applications, especially at high frequencies.

2.3 Noise-Equivalent Power (NEP) For MiXiNG. - Neglecting the noise from the LO and signal circuit $\left({ }^{2}\right)$ and assuming an ideal (noise-free) IF amplifier, we obtain a lower limit for the $N E P$ by setting the noise power $P_{\mathrm{N}}$ from the junction in the IF bandwidth equal to the IF signal power, namely $P_{\mathrm{IF}}=P_{\mathrm{N}}$. Assuming that we have only Johnson noise $P_{\mathrm{N}}$ from the shunt resistance and neglecting the noise contribution at $\omega_{\mathrm{IF}}$ which stems from beating of the noise at $\omega_{\mathrm{LO}} \pm \omega_{\mathrm{IF}}$ with the LO, then at $\omega_{\mathrm{IF}}, P_{\mathrm{N}}=k T B_{\mathrm{IF}}$, where $B_{\mathrm{IF}}$ is the IF bandwidth. The NEP is that signal power for which $P_{\mathrm{IF}}=P_{\mathrm{N}}$ at $\omega_{\mathrm{IF}}$. Using the fact that conversion efficiency $C_{\mathrm{e}}=P_{\mathrm{IF}} / P_{\mathrm{s}}$ we obtain, per cycle of bandwidth $\left({ }^{3}\right)$ :

$$
N E P=\frac{k T}{C_{\mathrm{e}}}
$$

for the mixer.

2.4 Dynamic Range (Linearity). - We define the dynamic range $D$ as the ratio of signal powers for which the IF response deviates from the linear response by $3 \mathrm{~dB}$, i. e.,

$$
D=\frac{\left(P_{\mathrm{s}}\right)_{\max }}{\left(P_{\mathrm{s}}\right)_{\min }}=\frac{\left(P_{\mathrm{s}}\right)_{\max }}{(N E P) B_{\mathrm{IF}}}
$$

where $\left(P_{\mathrm{s}}\right)_{\max }$ is the lowest signal power which gives a $3 \mathrm{~dB}$ deviation in the IF response from the linear response and $N E P$ is the noise equivalent power. The range of the signal current $I_{\mathrm{s}}$ for which we get IF output for a constant current bias is given by the change in the RF current that shifts the dc voltage from zero to $\hbar \omega_{\mathrm{LO}} / 2 \mathrm{e}$. Thus, the dynamic range for a cons-

(2) The LO and signal noise can be reduced by limiting the bandwidth of the coupling structure that couples the RF to the weak link. The LO noise can be further reduced by using a balanced mixer arrangement.

(3) The NEP of a receiver system consisting of mixer, IF amplifier, and video detector will depend on the bandwidth of the IF amplifier and the postdetection bandwidth of the squarelaw detector; the units for the $N E P$ will then be $\mathrm{W} / \sqrt{\mathrm{Hz}}$. tant current bias becomes extremely small for small $\Omega_{\mathrm{s}}$. It would therefore be advantageous to use a constant voltage bias.

Assuming that the dynamic resistance at this point in the $I-V$ characteristic is independent of the signal power, we find the linear range of the IF response for constant voltage bias from the linear range of the $I_{c}$ vs $I_{\mathrm{RF}}$ curve for the particular normalized signal frequency used. We assume a LO current amplitude $I_{\text {LO }}$ equal to $\frac{1}{2}$ of the value $I_{\text {RFo }}$ required to decrease the critical current to zero (Fig. 7). This is the value of the LO current which gives maximum conversion efficiency. The signal current $\left(I_{\mathrm{s}}\right)_{\max }$ corresponding to $\left(P_{\mathrm{s}}\right)_{\max }$ is given by the change in $I_{L O}$ for which the change in the critical current deviates by $3 \mathrm{~dB}$ from the dashed straight line in figure 7 . One can show that

$$
\frac{\left(I_{\mathrm{s}}\right)_{\max }}{I_{\mathrm{c}}} \approx \frac{1}{2} \frac{I_{\mathrm{RFO}}}{I_{\mathrm{c}}}
$$

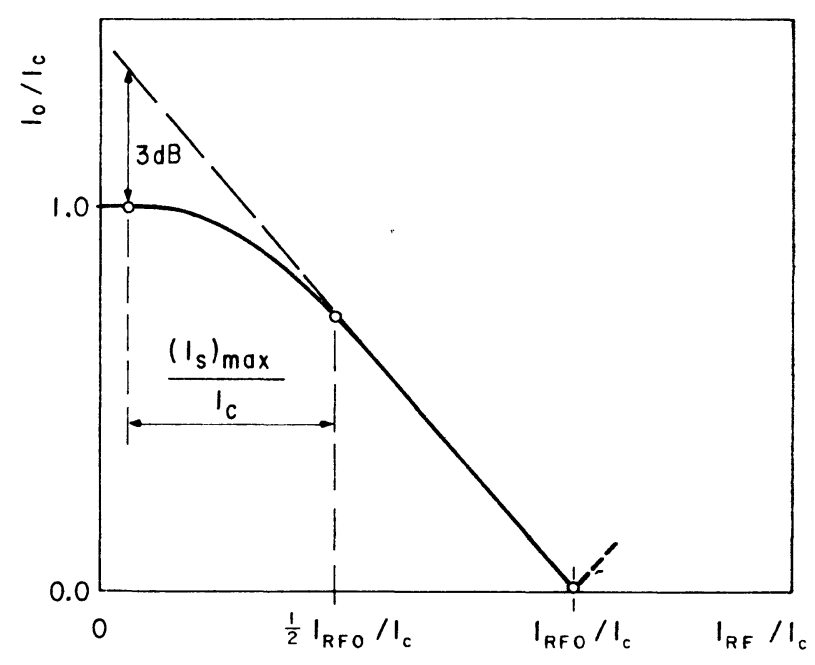

Fig. 7. - Maximum signal amplitude which gives a $3 \mathrm{~dB}$ deviation from the linear IF response.

for all $\Omega_{\mathrm{RF}}$. For low normalized frequency we find $I_{\mathrm{RFO}} / I_{\mathrm{c}} \approx 1$ and thus $\left(I_{\mathrm{s}}\right)_{\max } \approx \frac{1}{2} I_{\mathrm{c}}$. If we assume that the signal impedance of the weak link for this signal amplitude is $R_{\mathrm{s}} \approx R$, then the dynamic range in $\mathrm{dB}$ is given by

$$
D \lesssim 10 \log \frac{\frac{1}{2}\left(I_{\mathrm{c}} / 2\right)^{2} R}{(N E P) B_{\mathrm{IF}}}
$$

or with (5)

$$
D \lesssim 10 \log \frac{\left(I_{\mathrm{c}}^{2} / 8\right) R}{k T B_{\mathrm{IF}}} C_{\mathrm{e}} .
$$

It should be kept in mind, however, that the dynamic resistance does decrease as the signal power is increased, especially when it was high for small signal power (at low normalized signal frequencies); thus, the dynamic range is reduced.

2.5 Matching The Signal SOURCE. - Most Josephson devices have a low impedance compared to typi- 
cal RF source impedances. However, if one tries to increase $R$ (e. g. by choosing a proper geometry for constrictions) he runs into two limits because $I_{\mathrm{c}} R$ is constant for a given material and temperature : As $R$ is increased the noise increases and, in the case of low $\Omega_{\mathrm{s}}$, the dynamic resistance decreases - thus, also the conversion efficiency. In the case of high normalized frequencies, the effect of noise on $R_{\mathrm{dyn}}$ is negligible; however, the shunting effect of the intrinsic device capacitance is proportional to $\omega_{\mathrm{s}} C / G=\omega_{\mathrm{s}} \mathrm{RC}$ and thus increases with $R$. In addition, the capacitance parameter $\beta_{\mathrm{c}}=\omega_{\mathrm{c}} \mathrm{RC}$ is proportional to $R$ (independent of $\Omega_{\mathrm{s}}$ ) and hysteresis (which, for a sinusoidal $I-\Phi$ relation, is appreciable for $\beta_{\mathrm{c}}>0.7$ ) is increased for large $R$. One has therefore to compromise between matching losses and the adverse effects of high $R$ on the performance of the mixer.

3. Experimental results. - $3.1 I-V$ CHARACTERISTICS IN PRESENCE OF EXTERNAL RF. - All constrictions showed RF induced step structure, at least near the transition temperature, and most constrictions showed a weak Dayem effect [2] within a few millidegrees of the transition temperature $\left({ }^{4}\right)$. Constrictions with large dimensions $(\sim 1.0 \mu$ long, usually also $\sim 1 \mu$ wide) showed only a weak step structure near $T_{\mathrm{c}}$. The height of the zero-voltage step $I_{0}$ of these constrictions usually showed first a continuous decrease with increasing RF power and then a sudden drop to zero ; this drop became more pronounced for lower temperatures. Figure 8 shows a plot of $I_{0} / I_{\mathrm{c}}$ versus $I_{\mathrm{RF}}$ for a large constriction at $T=3.31 \mathrm{~K}$. These constrictions usually showed hysteresis in the $I-V$

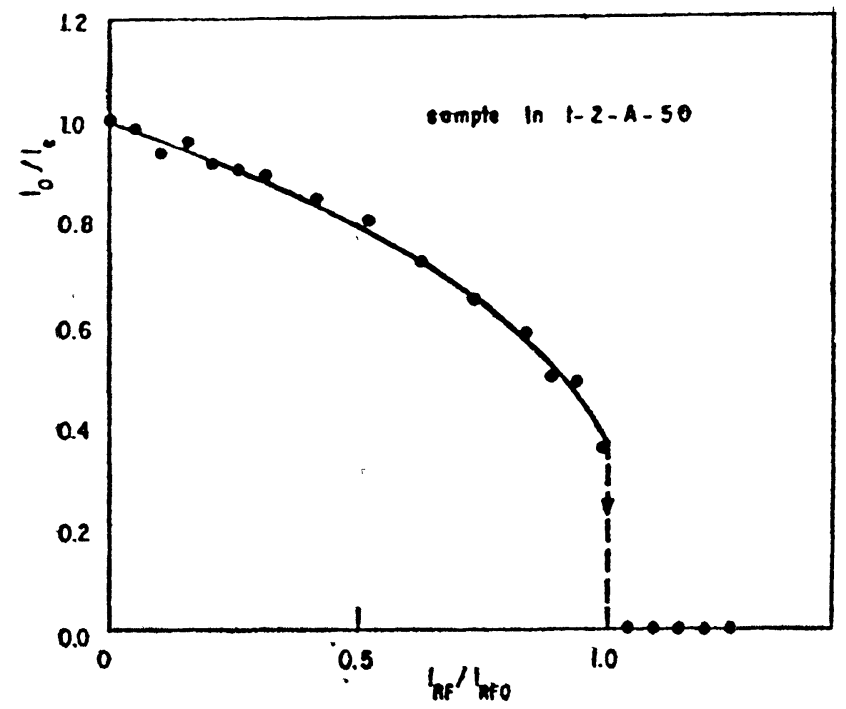

FIG. 8. - Height of zero-voltage step (normalized with respect to $I_{c}$ ) versus normalized RF current amplitude for fairly large constriction $(L, W \gtrsim 0.5 \mu)$ at $T \sim 3.3 \mathrm{~K}$.

(4) One test at $70 \mathrm{GHz}$ showed that the Dayem effect becomes much stronger at higher frequencies. This fact may be of interest for very high frequency detection. characteristic even near the transition temperature. There was also an observed hysteresis in the dependence of $I_{0}$ on RF power, suggesting the presence of thermal effects.

Short constrictions with $L \sim 1000-3000 \AA$ and $L / W \sim 0.3-1.3$ usually showed subharmonic structure for $I_{\mathrm{RF}} \gtrsim 0.5 I_{\mathrm{RFO}}$ even near $T_{\mathrm{c}}$. At lower temperatures or when they were very short and had $L / W \lesssim 0.4$, they showed strong subharmonic structure, sometimes even for fairly low RF currents $\left(I_{\mathrm{RF}} \gtrsim 0.2 I_{\mathrm{RFO}}\right)$. The latter also sometimes showed a low dynamic resistance and sometimes switching between the subharmonic steps. Constrictions with $L \lesssim 0.5 \mu$ and $W \lesssim 0.5 \mu$ usually showed some RF induced step structure even at the lowest temperature at which we tested them $(\sim 2.5 \mathrm{~K})$. Figure 9 shows the step structure of one of our best (and smallest) constrictions at $T=3.0 \mathrm{~K}$.

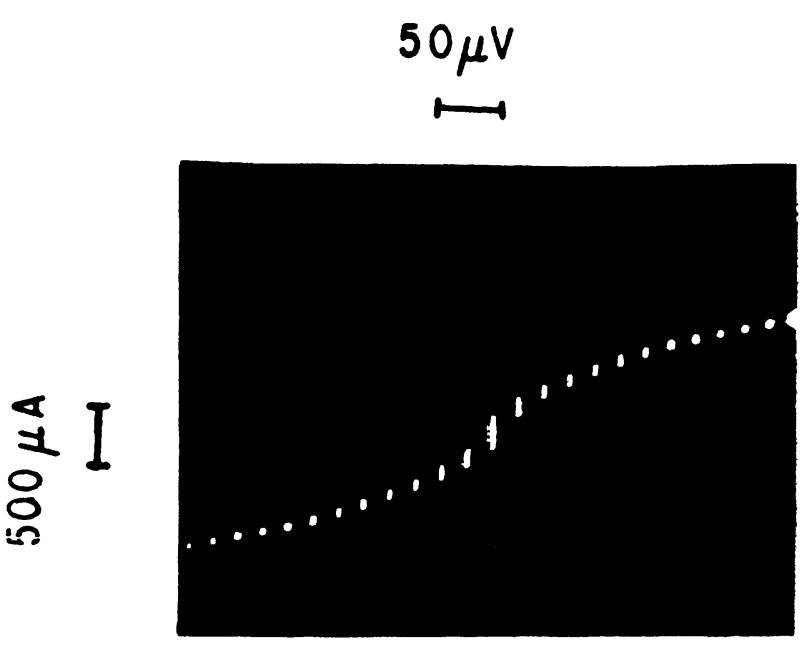

FIG. 9. $-I-V$ characteristic in presence of RF $(\sim 10 \mathrm{GHz})$ for small constriction $(L \approx 0.32, L / W \approx 0.97,1200 \AA$ thick), at $3.0 \mathrm{~K}$. The 12 th harmonic step is still clearly visible.

Oscillation of the step heights with RF power was only observed in a few constrictions which had $L \approx 2000-4000 \AA$ and $L / W \approx 0.7-1.0$. The dependence of the heights of the 0 th and 1st steps on $I_{\mathrm{RF}} / I_{\mathrm{RFO}}$ are plotted in figure $10 a$.

A quick method of finding the normalized frequency for a device satisfying the resistively shunted junction model is to find and evaluate the ratios of power required to drive the various steps to zero. Curves of these ratios can be plotted from the model as in figure $11 a$. The triangular points represent values of the power ratios for the indicated steps. Here we could quickly see that $\Omega_{\mathrm{RF}} \approx 0.46$. Having found $\Omega_{\mathrm{RF}}$ one can find the normalized RF current $i_{\mathrm{RFO}}=I_{\mathrm{RFO}} / I_{\mathrm{c}}$ that depresses the critical current to zero from figure $11 b\left({ }^{5}\right)$. Most of our constrictions showed,

(5) The value of $i_{\mathrm{RFO}}$ is needed for the calculation of the required LO power from figure $6 b$, as we saw in Section 2.2. 


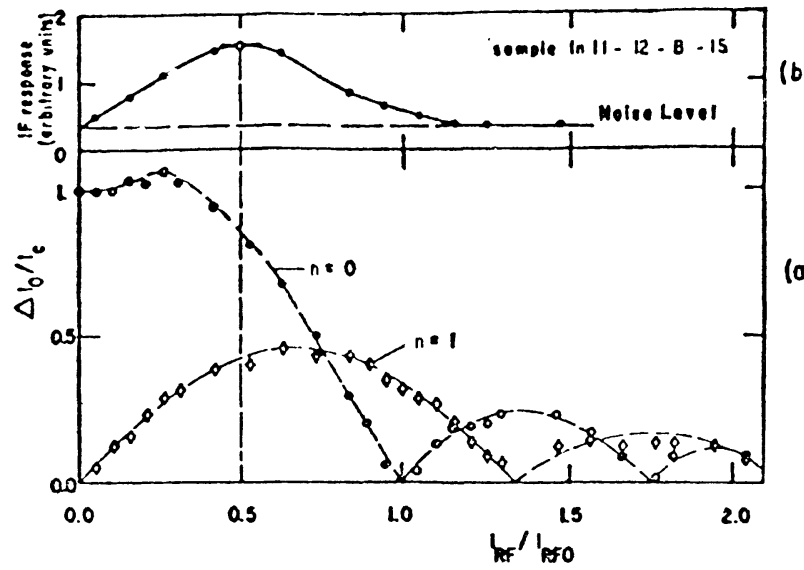

FIG. 10. - (a) Normalized step height of 0th and 1st RF induced steps as a function of RF current amplitude. (b) The corresponding IF response as a function of $I_{\mathrm{LO}}=I_{\mathrm{RF}}$ when an additional small signal is applied. The maximum IF response occurs at a $\mathrm{LO}$ current value $I_{\mathrm{LO}} \approx \frac{1}{2} I_{\mathrm{RFO}}$.

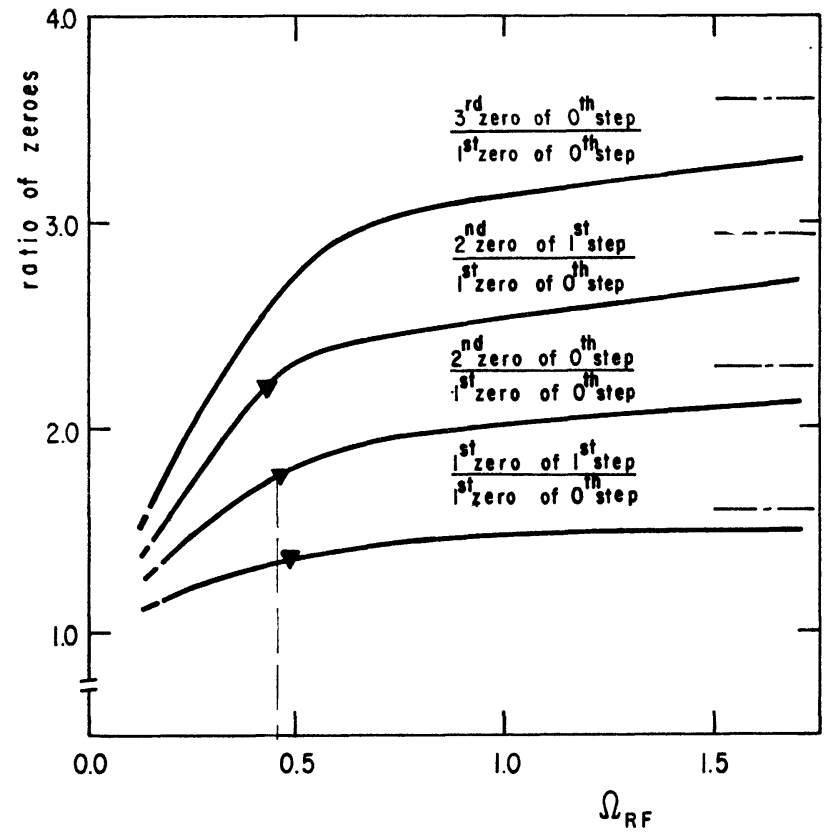

FIG. $11 a$. - Calculated current amplitude ratios of zeros of the 0 th and 1st induced steps as a function of the normalized frequency $\Omega_{\mathrm{RF}}$.

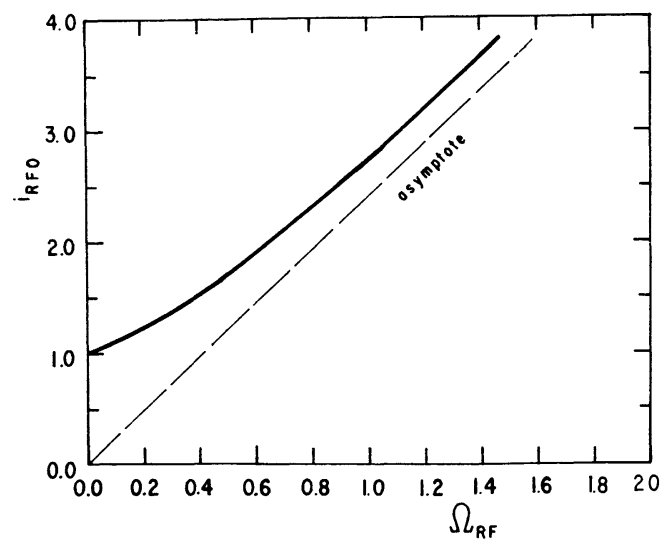

FIG. 11b. - Calculated normalized RF current amplitude $i_{\mathrm{RFO}}=I_{\mathrm{RFO}} / I_{\mathrm{c}}$ which depresses the critical current to zero versus normalized RF frequency $\Omega_{\mathrm{RF}}=\hbar \omega_{\mathrm{RF}} / 2 \mathrm{e} I_{\mathrm{c}} R$. however, much less agreement with the RSJ model, especially at low temperature.

3.2 Mixing EXPERIMENTS. - We applied a signal at frequency $\omega_{\mathrm{s}} / 2 \pi \approx 9.75 \mathrm{GHz}$ and a LO separated from the signal by the IF frequency $\omega_{\mathrm{IF}} / 2 \pi$ of $30 \mathrm{MHz}$; both microwave oscillators were cavity stabilized. Our experiments clearly fulfilled the assumption of low $\omega_{\mathrm{IF}} / \omega_{\mathrm{s}}$.

A close relation between the $I-V$ characteristics in the presence of the LO alone and the IF response in the presence of an additional small signal was observed. As expected from the theory, the IF response for small signals occurred between the LO induced steps. The maximum IF response always occurred between the 0th and 1st LO steps for $T$ not too far below $T_{\mathrm{c}}$, and increased with increasing dynamic resistance between steps. The maximum IF response always occurred for $I_{\mathrm{LO}}$ between 0.4 and 0.6 of $I_{\mathrm{RFO}}$, the value needed to reduce $I_{0}$ to zero (see Fig. 6). Upon reducing the temperature or increasing the LO current, however, biasing between the two lowest steps became impossible and the IF response disappeared completely in that region. The IF response could then be seen above the 1st step ; however, as expected the sensitivity was lower. Further increases of $I_{\mathrm{LO}}$ eliminated IF response at successively higher steps because of switching between steps. This was observed at $\approx 3.2 \mathrm{~K}$.

Probably one could still operate the constriction between the 0th and 1st steps even at low temperature if a constant voltage bias were used, also one could probably use this temperature range even for a dc current bias if the applied frequency was higher (a higher normalized frequency $\Omega_{\mathrm{s}}$ gives a lower dynamic resistance).

3.2.1 Influence of constriction dimensions on IF response. - The dependence of the IF response on the dimensions follows from the above description of the $I-V$ characteristics in the presence of RF radiation. Large constrictions and those with low dynamic resistance between steps (e. g., very short constrictions which showed a low $R_{\mathrm{dyn}}$ due to many rounded subharmonic steps) showed poor IF response. Constrictions with hysteresis showed a low IF response between the LO steps on the lower branch of the hysteresis loop. The best performance was achieved with those small constrictions that showed higher periods of the RF induced steps.

3.2.2 Influence of temperature on IF response. The theory predicted an increase of the IF response (and, therefore, conversion efficiency) for decreasing normalized signal frequency $\Omega_{\mathrm{s}}$ due to an increased dynamic resistance between steps and a steeper slope of the $I_{\mathrm{c}}$ versus $I_{\mathrm{RF}}$ curve. The shunt resistance $R$ of the constrictions is approximately temperature independent as deduced from experimental evidence, but $I_{\mathrm{c}}$ increases with decreasing $T$, so the normalized frequency $\Omega_{\mathrm{s}}=\hbar \omega_{\mathrm{s}} / 2 \mathrm{e} I_{\mathrm{c}} R$ decreases with decreasing 
$\boldsymbol{T}$. However, the agreement with the RSJ model became worse at lower temperature ; in particular, the decrease of the critical current with $I_{\mathrm{RF}}$ never approached the nearly straight line which the RSJ model predicts for small $\Omega_{\mathrm{RF}}$. Moreover, many constrictions showed the above-described switching between steps or hysteretic $I-V$ characteristics. Nevertheless, the constrictions showed first a rapid increase in the IF response with decreasing temperature as the noise rounding of the LO steps decreased and thus the dynamic resistance between steps increased. Small constrictions showed a further increase in the IF response with decreasing temperature until the IF response between the 0th and 1st steps suddenly disappeared due to the above-mentioned switching.

3.2.3 Sensitivity. - We measured the sensitivity of the devices for mixing by adjusting the signal power level such that the output of the $30 \mathrm{MHz}$ receiver was twice the noise level. This signal power is the minimum detectable power which, for a good but unmatched device was -60 to $-65 \mathrm{dBm}$.

We give now an estimate of how much power was actually coupled into the constriction for a typical experiment: The constriction used in one typical experiment had a critical current $I_{\mathrm{c}}$ of $245 \mu \mathrm{A}$ at $T=3.28 \mathrm{~K}$ and an asymptotic resistance $R \approx 0.28 \Omega$. The RF frequency was $9.75 \mathrm{GHz}$, the minimum detectable power was $-64 \mathrm{dBm}$. From $I_{\mathrm{c}}, R$ and $\omega_{\mathrm{RF}}$ we find

$$
\Omega_{\mathrm{RF}}=\frac{\hbar \omega_{\mathrm{RF}}}{2 \mathrm{e} I_{\mathrm{c}} R} \approx 0.29
$$

(The agreement with the RSJ model was not very good ; the best fit was obtained for $\Omega_{\mathrm{RF}} \approx 0.15 \pm 0.05$.) For this range of normalized frequencies (0.1-0.3) the value of the RF current which depresses $I_{\mathrm{c}}$ to zero is $I_{\mathrm{RFO}} / I_{\mathrm{c}} \approx 1.27 \pm 0.13$, thus $I_{\mathrm{RFO}} \approx 310 \mu \mathrm{A}$. The dissipated RF power for this RF level should then be of the order of

$$
P_{\mathrm{RF}} \sim \frac{I_{\mathrm{RFO}}^{2} R}{2}=13.5 \times 10^{-9} \mathrm{~W} .
$$

The measured RF power input $P_{\text {RFo }}$ that depresses $I_{\mathrm{c}}$ to zero was $27.6 \mu \mathrm{W}$. Thus only about $0.05 \%$ of the incident power was coupled into the constriction. An independent check of the order of magnitude can be found as follows. As the impedance of the constriction was very small compared to the waveguide impedance, the RF current $I_{\mathrm{RF}}$ in the constriction should be of the order of $I_{\mathrm{RF}} \sim V_{\mathrm{RF}} / Z_{0}$ where $V_{\mathrm{RF}}$ is the RF voltage of the equivalent RF source. In terms of the available power $P_{\mathrm{a}}=V_{\mathrm{RF}}^{2} / 8 Z_{0}$ from the RF source we find $I_{\mathrm{RF}}=\sqrt{8 P_{\mathrm{a}} / Z_{0}}$. Using the characteristic impedance

$$
Z_{0}=\frac{377}{\sqrt{1-\left(f_{\mathrm{c}} / f\right)^{2}}}
$$

where $f_{\mathrm{c}}$ is the cut-off frequency of the waveguide, we find $Z_{0} \approx 430 \Omega$. Using the above value of $P_{\mathrm{RFO}}$ for $P_{\mathrm{a}}$, we find

$$
I_{\mathrm{RFO}}=\sqrt{\frac{8 P_{\mathrm{RFO}}}{Z_{0}}} \approx 720 \mu \mathrm{A},
$$

which is in reasonable agreement with the above found value. We estimate therefore that the minimum detectable power should be $\sim 10 \log 200=40 \mathrm{~dB}$ better for a matched signal (assuming a loss-free matching network). In addition, we had a mismatch in the IF circuit and an additional loss due to a high contact resistance of $\approx 50 \Omega$ between the constriction and the IF circuit leads. We estimated $R_{\mathrm{dyn}} \approx 4 \Omega$, thus with an additional series loss resistance (contact resistance) of $50 \Omega$ and an IF amplifier input impedance of $50 \Omega$, we find that only $\sim 8 \%$ of the available IF power is coupled into the IF amplifier. This is an additional loss of $10 \log 12.5=11 \mathrm{~dB}$. We estimate therefore a minimum detectable power of $-118 \mathrm{dBm}$ for matched signal and IF impedance. The IF bandwidth was $\approx 2 \mathrm{MHz}$ and the postdetection bandwidth $12.5 \mathrm{~Hz}$, the measured $N E P$ for 1 cycle post detection bandwidth was therefore $8 \times 10^{-14} \mathrm{~W} / \sqrt{\mathrm{Hz}}$ and the estimated $N E P$ for the matched case would be $N E P \approx 10^{-18} \mathrm{~W} / \sqrt{\mathrm{Hz}}$, which is better than the best room-temperature mixers. The NEP is in this case limited by the room temperature IF amplifier.

3.2.4 Conversion efficiency. - We measured the conversion efficiency $C_{\mathrm{e}}$ for the unmatched case to be $-36 \mathrm{~dB}$, which was a typical number for good devices. With the above estimated improvement for matched conditions, the conversion efficiency would increase to $\approx+15 \mathrm{~dB}$, i. e., conversion gain. From the theory, one would also expect conversion gain for the low normalized frequency used.

3.2.5 Linearity of IF response. - For low signal power, the IF response is linear. For larger signal power, the $I-V$ characteristic is changed by the signal power, causing a decrease in $R_{\mathrm{dyn}}$ and thus a saturation of the IF response. For still higher signal power the LO steps become essentially washed out and both the $I-V$ characteristic and the IF response become fairly complicated. When the constriction is operated in a region of high dynamic resistance (which is desirable to achieve high sensitivity and conversion efficiency), very small signal amplitudes cause a decrease of $R_{\mathrm{dyn}}$ and thus a nonlinear response. Figure 12 shows a typical plot for the IF response versus signal amplitude. We found that a signal current in the range $(0.01-0.06) I_{\text {RFo }}$ caused a sufficient change of $R_{\text {dyn }}$ to lower the IF response by $3 \mathrm{~dB}$ below the linear range; the lower value of $I_{\mathrm{s}}$ being for high $R_{\text {dyn }}$ usually achieved at lower $T$ (lower $\Omega_{\mathrm{s}}$ ), the larger value for small $R_{\mathrm{dyn}}$ at higher $T$ (higher $\Omega_{\mathrm{s}}$ ).

The dynamic range $D$, as defined earlier, for this 


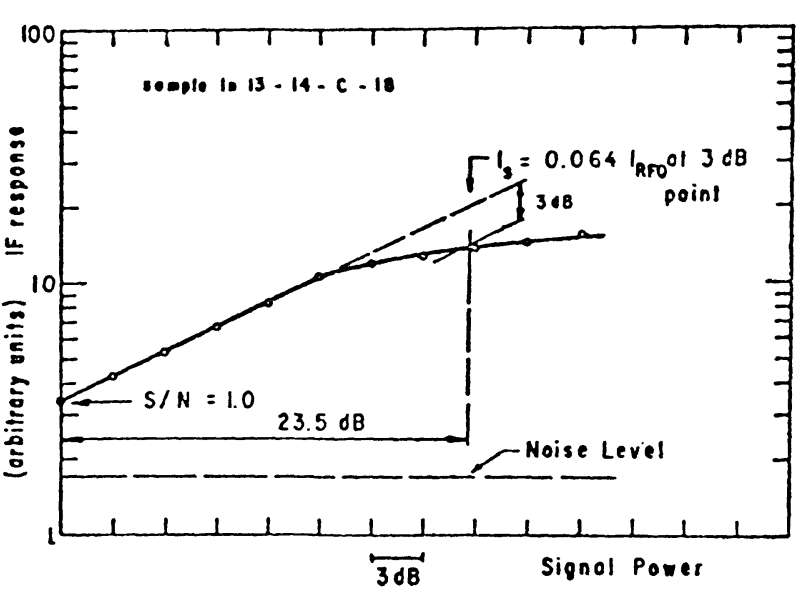

FIG. 12. - Typical IF response versus signal power. The dynamic range in this case was $23.5 \mathrm{~dB}$. The signal current corresponding to the $3 \mathrm{~dB}$ point in the figure, was $6.4 \%$ of the RF current amplitude that depressed the critical current to zero.

experiment was only $D \approx 23.5 \mathrm{~dB}$. However, if the device were matched, the $N E P$ would decrease and thus the dynamic range increase by the same amount; i. e., for the above estimate we would expect a dynamic range of $D \approx 74.5 \mathrm{~dB}$.

4. Conclusion. - The calculations made on the resistively shunted junction model are in substantial agreement with experiment and can be used as a guide for the design of low-capacitance Josephson device mixers. The calculations show that mixing with gain is possible even in the presence of noise and that extremely low LO power is required for this type of mixer (typically $10^{-9} \mathrm{~W}$ ). The low LO power may be important for very high frequency applications or when whole arrays of weak links should be used. Moreover the noise contribution from the LO plays then a much less crucial role possibly eliminating the need of balanced mixers.

We have fabricated indium thin-film constrictions which have dimensions within a factor of two of the smallest achievable by electron-beam lithography. Our measured performance figures suffered from RF and IF mismatches. Corrections for the mismatches lead to estimates of NEP and conversion gain as good as the best point contacts.

These film constrictions made of soft metals like indium are very susceptible to burn-out. Constrictions of tantalum, niobium or vanadium would be more robust though the lower coherence length may require unachievably small dimensions to get performance comparable with soft-metal constrictions. More experimental evaluation is needed for both kinds to determine parametric dependences of the mixing properties.

\section{References}

[1] Grimes, C. C. and Shapiro, S., Phys. Rev. 169 (1968) 397.

[2] Anderson, P. W. and Dayem, A. H., Phys. Rev. Lett. 13 (1964) 195.

[3] Dayem, A. H. and Wiegand, J. J., Phys. Rev. 155 (1967) 419.

[4] Gregers-Hansen, P. E. and Levinsen, M. T., Phys. Rev. Lett. 27 (1971) 847.

[5] Russer, P., J. Appl. Phys. 43 (1972) 2008.
[6] Laibowitz, R., Revue Phys. Appl. 9 (1974) 165.

[7] Auracher, F. and Van Duzer, T., Proc. 1972 Applied Superconductivity Conference, Annapolis, Md. USA, 1-3 May 1972.

[8] Gregers-Hansen, P. E., Levinsen, M. T. and Pedersen, G. F., J. Low Temp. Phys. 17 (1972) 99.

[9] TAUR, Y. et al., Revue Phys. Appl., 9 (1974) 263. 